\title{
ASTROMETRY IN CHINA
}

Ye Shu-hua

Shanghai Observatory, Academia Sinica, China

The space age and modern technology have opened a new era for astrometry. A great amount of modern, precise astrometric data and approaches is urgently needed not only for astrophysics but also for Earth Science research. The Astrometric Community looks forward to establishing a precisely specified uniform celestial frame of reference for all purposes, which will link the bright stars, faint stars, and galaxies, down to the remote radio sources, and to establish a terrestrial frame of reference as well as to specify a precise transformation between these two frames. Effective international cooperation is the goal of all astrometrists. The Chinese astronomers are ready to join and play an active part in this cooperation.

Astrometry is therefore one of the important branches of astronomy in China. Six observatories are devoting their efforts to classical astrometry, including star catalogues, positions of major planets and radio stars, and determination of the parameters of the Earth's rotation (ERP). Photographic astrometric work has been carried out on proper motions in star clusters, star associations, RR Lyrae variables, on positions of stars and minor planets, as well as on parallaxes and on occultations. Recently, developments of new techniques such as laser ranging and VLBI have pushed astronomers to consider more deeply the frame of reference coordinates. Work in this field is also being done.

\section{The observatories}

The location, epoch where astrometric work started, facilities and research topics are as follows. This is a list of the instruments active at the various observatories (the longitude are east longitude, the latitudes are positive).

1. Shanghai Observatory $(1951-\quad), 120^{\circ} 26^{\prime}, 31^{\circ} 12^{\prime}$

Semiautomatic photoelectric transit instrument

Photoelectric astrolabe (Type II) $20 \mathrm{~cm}$

Danjon astrolabe (No. 14)
ERP, star catalogues, position of radio stars and major planets 
Doppler receiver

SLR system $(D=60 \mathrm{~cm}, \mathrm{YAG}$ laser $)$

$40 \mathrm{~cm}$ refractor $(\mathrm{f}=7 \mathrm{~m})$

* $1.56 \mathrm{~m}$ reflector $(\mathrm{f}=1.56 \mathrm{~m}$, under construction)

$6 \mathrm{~m}$ radio telescope $(\lambda>6 \mathrm{~cm}, \mathrm{MKII}, \mathrm{VLBI}$,

* $25 \mathrm{~m}$ radio telescope

( $\lambda>1.3 \mathrm{~cm}$, MKIII, VLBI, under construction)
ERP, space geodesy ERP, space geodesy, geodynamics

proper motions, occultations, positions of stars and of minor planets

parallaxes, positions,

ERP, geodynamics, radio astrometry

ERP, geodynamics, radio astrometry

2. Purple Mountain Observatory, Nanjing (1952 - I), $118049^{\prime}, 32004^{\prime}$

Automatic photoelectric transit

$60 \mathrm{~cm}$ reflector $\mathrm{f} / 9$

$40 \mathrm{~cm}$ refractor $\mathrm{f}=3 \mathrm{~m}$

* Photoelectric Transit (in planning stage), $40 \mathrm{~cm}$

Doppler receiver
ERP, star catalogues

minor planets, optical position of radio sources, comets

star catalogues

ERP, space geodesy

3. Beijing Observatory (1959- ), 116020', 400 06'

Photoelectric transit

Photoelectric astrolabe (Type II) $20 \mathrm{~cm}$

Danjan astrolabe (No. 30)

ERP, star catalogues, positions of radio stars and major planets

SLR system $(D=40 \mathrm{~cm})$

$60 \mathrm{~cm}$ reflector $\mathrm{f} / 9$

* Photoelectric astrolabe, $30 \mathrm{~cm}$

(Type III, under construction)

ERP, space geodesy occultations

star catalogues

Tianjin Latitude Station (1957- ) ), 117003', 390 08'

ZTL-180

PZT $26 \mathrm{~cm}$

ERP, star catalogues

Doppler receiver MX-1502

ERP, space geodesy 
4. Wucheng Time Station, Institute of Geodesy and Geophysics, Wuhan, (1963 ), $114^{\circ} 21^{\prime}, 30^{\circ} 32^{\prime}$

Photoelectric transit

Danjon astrolabe (No. 29)

ERP, star catalogues

5. Shaanxi Observatory (1968 - ) near Xian, $109^{\circ} 33^{\prime}, 34^{\circ} 57^{\prime}$

Semiautomatic photoelectric transit

Photoelectric astrolabe (Type I) $15 \mathrm{~cm}$

* PZT (under construction) $26 \mathrm{~cm}$

ERP, star catalogues

6. Yunnan Observatory, Junming (1973 - ), $102^{\circ} 47^{\prime}, 2^{\circ} 02^{\prime}$

Photoelectric transit (experimental observation)

Photoelectric astrolabe (Type II) $20 \mathrm{~cm}$

ERP, star catalogue

* Transit Circle for low latitude $(40 \mathrm{~cm}$;

(in planning stage)

star catalogues

SLR system $(D=40 \mathrm{~cm}, \mathrm{SGB})$

ERP, space geodesy, geodynamics

$10 \mathrm{~m}$ radio telescope $(\lambda>3 \mathrm{~cm}$, MKII VLBI)

Radio astrometry,

(under construction)

ERP, geodynamics

Doppler receiver

ERP, space geodesy

\section{Star Catalogue}

The catalogues listed below have been compiled from observations made in China on photoelectric transits, photoelectric astrolabes, Danjon astrolabes, ZTL180, etc. All the program stars were selected from the FK4, the FK4 sup., and the GC. $T$ is the observing period; $N$ the number of star and $\varepsilon$ denotes the standard error which characterize the precision of the position components in units of milliseconds of time in RA and milliarcseconds in declination.

1. Right Ascension Catalogues Observed on Transit Instruments

$\begin{array}{llll} & \mathrm{T} & \mathrm{N} & \varepsilon \\ \text { Shanghai } & \text { ( 1) } 1957-1964 & 410 & \\ & \text { ( 2) } 1966-1969 & 410 & 1.5 \\ & \text { (3) } 1972-1974 & 447 & \\ \text { (4) } 1964-1966 & 464 & 4.3 \\ \text { Purple Mountain } & \text { (5) } 1971 & 350 & 4.3 \\ \text { Beijing } & \text { (6) } 1966-1970 & 509 & \\ & \text { ( 7) } 1971-1976 & 509 & 2.0 \\ \text { Shaanxi } & \text { ( 8) } 1968-1972 & 324 & \\ & \text { (9) } 1979-1981 & 467 & 2.8 \\ \text { Wucheng } & (10) 1971-1973 & 407 & \\ & (11) 1975-1978 & 407 & \\ & \text { (12) } 1976-1979 & 464 & \end{array}$


Catalogues (4) and (5) were observed on Hainan Island (in the south of China). The observations for all the catalogues were carried out on a photoelectric transit, except for those in catalogue (4), which were obtained on a visual transit.

2. Catalogues observed on a Danjon Astrolabe

$\begin{array}{llcll} & T & N & \varepsilon_{\alpha} \cos \delta & \varepsilon \delta \\ \text { Shanghai } & \text { (1) } 1961-1964.5 & 92 & 1.8 & 24 \\ & \text { (2) } 1965-1972 & 92 & & \\ \text { Beijing } & \text { (3) } 1973-1978 & 92 & & \\ \text { Wucheng } & \text { (4) } 1967-1972 & 280 & 4.4 & 74 \\ & \text { (5) } 1965-1975 & 98 & 2.0-7.0 & 30-130\end{array}$

3. Star Catalogues observed on a Photoelectric Astrolabe

$\begin{array}{lllll} & T & N & \varepsilon_{\alpha} \cos \delta & \varepsilon_{\delta} \\ \text { Shaanxi } & \text { (1) } 1973-1976 & 108 & 2.2 & 34 \\ \text { Shanghi } & \text { (2) } 1973-1981 & 820 & 4.3 & 72 \\ & (3) 1975-1978 & 103 & 1.9 & 22 \\ \text { Beijing } & (4) 1979-1981 & 530 & 3.4 & 61 \\ & (5) 1976-1978 & 196 & 2.6 & 46 \\ \text { Yunnan } & (6) 1979-1981 & 906 & 3.5 & 54 \\ & (7) 1976-1978 & 182 & 3.3 & 56 \\ & \text { (8) } 1979-1981 & 284 & 2.2 & 38\end{array}$

4. Declination Catalogue observed on a ZTL-180 (Tianjin)

229 pairs of ILS program I-VI and 55 pairs of FZT were observed in 1963-1967, $\varepsilon_{\delta}=0 ! 029$.

5. Compiled Catalogues

(1) The Catalogue of Right Ascension for the Time Service of China (CTC) was compiled at the Shaanxi Observatory in 1974, from the right ascension catalogues observed in China available at this time, including 1156 stars with precisions corresponding to internal standard errors of 0.002 .

(2) The Catalogue of Right Ascension and Proper Motion of 904 Bright Stars was compiled at the Shanghai Observatory in 1976 from the five right ascension catalogues observed at this observatory, with $\varepsilon_{\alpha \cos } \delta=0.0025$, $\varepsilon_{\mu \alpha} \cos \delta=0.0144 / 100$ yrs.

(3) The Danjon Astrolabe Catalogue was compiled at the Shanghai Observatory in 1979, from three Danjon catalogues of this observatory, including 129 right ascension and 96 declinations as well as their proper motions. The precisions correspond to the following errors: 
$\varepsilon_{\alpha} \cos \delta=0.0016$
$\varepsilon_{\delta}=0.028$

$\varepsilon_{\mu} \cos \delta=0$ s $012 / 100$ yrs
$\varepsilon_{\mu}^{\prime}=0 ! 240 / 100$ yrs

(4) The Combined Astrolabe Catalogue (I) was compiled at the Beijing Observatory and the Astronomical Department of Nanjing University in 1980, incorporating all the Danjon astrolabe and photoelectric astrolabe catalogues obtained in China. It lists positions for 608 stars. Precisions measures: $\varepsilon_{\alpha} \cos \delta=$ $0.0032, \varepsilon_{\delta}=0.056$.

(5) The General Catalogue of Photoelectric Astrolabes (GCPA) was compiled in 1982 at the six observatories, based mainly on the new photoelectric astrolabe catalogues observed during the period 1978-1981. It covers 1579 stars, (with $\varepsilon_{\alpha} \cos \delta=0.033, \varepsilon_{\delta}=0.058$ ).

\section{Photographic Astrometry}

The long focus $40 \mathrm{~cm}$ astrograph at the Zo-Se Section of Shanghai Observatory was used mainly for photographic astrometry. There exist more than six thousand plates dating from the beginning of this century, including some of Halley's Comet taken in 1910. Proper motions and positions of stars, open clusters, and star associations, were obtained during the past 30 years.

Based on the Shanghai Observations and others obtained elsewhere, a catalogue of proper motions for 168 RR Lyrae variables was compiled, the spatial motions and absolute magnitudes of these stars - critical for the calibrations of the cosmic distance scale - have been investigated by using their proper motions and radial velocities.

From proper motion data, 375 cluster member stars have been identified in the region of the open cluster M67 (NGC 2862).

At the Purple Mountain Observatory, many studies on photographic positions of asteroids and comets were carried out. Several dozen new asteroids and three comets discovered there were subsequently named and numbered by the International Minor Planet Center. Precise positions of asteroids have been observed at the Shanghai Observatory mainly to contribute to the discussion of their orbits. Quingtao Observatory had observed asteroids for many years but was closed several years ago.

For the purpose of the determination of stellar diameters, observations of stellar occultation are carried out at the Beijing and Shanghai Observatories with the use of a fast photoelectric photometer.

The $40 \mathrm{~cm}$ astrograph was used for the derivation of absolute proper motion of stars with respect to galaxies in the zone from $-5^{\circ}$ to $-25^{\circ}$. The observations began in 1955, and the second epoch plates are now being exposed.

This astrograph was recently also used for experimental observations of trigonometric parallaxes. 
This instrument is also still used for micrometric observations of visual binaries. A list of 373 visual binaries, including 14 new pairs, has been published. The 1.56-meter astrometric reflector is now under construction at the Shanghai Observatory, and will be used mainly for the determination of trigonometric parallaxes and/or precise positions of nearby stars, such as dwarfs, cool carbon stars, QSO objects, etc..

For the Halley Watch, plans for precise photographic position measurements are under consideration.

\section{The Celestial Reference Frame}

The new definition of UT and the Celestial Reference Pole have been investigated mainly at the Department of Astronomy of Nanjing University. The influence of the newly adopted astronomical constants on the celestial reference frame are discussed at the Shanghai Observatory, at Purple Mountain Observatory, and at Nanjing University. Shaanxi Observatory investigated the correction to the aberration constant and the solar semiannual nutation term which appeared in the photoelectric astrolabe observations.

The 1961-1976 Danjon astrolabe observations yielded the estimate $9 " 197 \pm 0 ! 003$ for the principal nutation coefficient in an investigation carried out at the Shanghai Observatory. This astrolabe has been used to observe major planets for a long-term program, beginning in 1964 and again in 1973. The correction to the FK4 equinox from the observation deriving 1973-1980 results as $\Delta \mathrm{E}=0.044$ from observations of Mars and as 0.048 from observations of Jupiter. These estimates agree well with the results from other series of meridian observations.

Radio astrometry also is under serious consideration in China. Besides establishing several radio telescopes for this purpose, for example at the Shanghai Observatory, the Yunnan Observatory and elsewhere, the relationship between optical and radio reference frames has been studied. Identification of the optical counterparts of radio sources and their precise position are being investigated at the Purple Mountain and Shanghai Observatories. Meanwhile, bright radio stars are being observed on the Danjon astrolabe, a photoelectric astrolabe and a photoelectric transit, at the Shanghai and Beijing Observatories to compare the FK4 directly with the radio catalogue.

\section{Earth Rotation Parameters}

The six observatories join in the national network of UT and polar motion services, which have been operating continiously for many years. Recently, the six observatories have started to participate in the BIH and IPMS service as well as the MERIT Campaign.

For the MERIT main campaign, thirteen classical instruments, including four photoelectric astrolabes, two Danjon astrolabes, one PZT, one ZTL-180 and five photoelectric transits are used to gather observations. One of the two Doppler receivers (MX-1502) is located at Shanghai and is jointly operated by the Research Institute of the National Bureau of Surveying and Mapping and the Shanghai 
Observatory; the other one is located at Nanjing, and operated by the Purple Mountain and Shaanxi Observatories and Tien-jin Station. The LAGEOS tracking station of the Shanghai Observatory also participates in the campaign. World data on classical, SLR, LLR, VLBI, and Doppler observations are to be analyzed in China, mainly at the Shanghai and the Purple Mountain Observatories.

A VLBI station equipped with a 25-meter radio telescope and a MK III terminal is going to be established at Shanghai. At present, a 6-meter radio telescope with a MK II terminal is being used for experimental VLBI in cooperation with some observatories. The 10-meter radio telescope at the Yunnan Observatory will also take part in VLBI observations. Research on the characteristics, the periodicities, and the mechanism of Earth rotation and polar motion has been carried out at the six observatories. The secular polar motion and secular variation of the Earth's rotation, the power spectrum of and periodicity in the Earth's rotation and polar motion and the correlation between these variations and large earthquakes, the angular momentum of the atmosphere, the effects of Earth tides and ocean load on the observed UT and latitude, local variation on longitude and latitude etc., have been and will continue to be investigated in China.

\section{Improvements of Method and Instrumentation}

In China, we had already modified the visual transits to photoelectric ones, and more recently, succeeded with automation at the Purple Mountain Observatory by means of a minicomputer and several mechanical devices. Meanwhile, Shanghai and Shaanzi Observatories both changed their transits to a semiautomatic mode. Automatic level reading has been accomplished at the Wucheng Station. Beijing Observatory is changing the mechanical contact of the instruments to photoelectric ones.

In the early 1970's, the Astronomical Instrument Factory at Nanjing, in cooperation with the Beijing and the Shanghai Observatories designed and built our photoelectric astrolabe. There are four of them in operation now. Also, one PZT has been built and is in operation at Tien-jin. The other two are under construction.

Beijing Observatory is converting its Danjon astrolabe into a two-prism instrument, and the Shanghai Observatory is going to make the same modification after the MERIT Campaign.

After a long discussion about the advantage of the equal-altitude method for star catalogue observation, a new photoelectric astrolabe (Type III) is being built for this purpose and will observe stars down to 11th magnitude. The new astrolabe catalogue project is being lead by the Beijing Observatory. The Yunnan Observatory, because of its low latitude $\left(25^{\circ}\right)$, proposes to build a photoelectric Meridian Instrument which would determine the azimuth by observing stars on a great circle perpendicular to the Meridian, to give an absolute catalogue at low latitude. Experimental observations carried out on a normal photoelectric transit proved that the observed azimuths are sufficiently precise for this purpose. 
A precise, absolute catalogue down to magnitude 11 will thus appear during the next decade and will be a good tool for the equal-altitude and the meridian mode of observation.

The 1.56-meter reflector will be the highlight of photographic astrometry in China. This instrument has been designed and is being built in Shanghai, mainly to be used for the determination of parallaxes of nearby stars.

The 25-meter radio telescope now being built for Shanghai Observatory will become an active instrument of the world VLBI network for astrometry, astrophysics, geodesy, and geodynamics.

Discussion:

MURRAY: Is your catalogue of RL Lyrae variables available?

YE: We have 168 stars, almost ready for publication.

GUINOT: Concerning the Earth's rotation, I want to mention that the contribution of Chinese astronomers is important not only because of the number of measuring instruments, but also because of the high quality of the results of each of these instruments. This is especially important for UT1 because up to nowr, the results of new methods are not obtained on a truly operational basis, with the exception of connected element radio interferometry. 